\title{
An Immersed FEM for Elasticity Equations with Interfaces
}

\author{
Zhilin $\mathrm{Li}^{*} \quad$ Xingzhou Yang ${ }^{\dagger}$
}

August 12, 2004

\begin{abstract}
The immersed finite element method based on a uniform Cartesian mesh has been developed for elasticity equations with discontinuous physical parameters across an interface in this paper. The interface does not have to be aligned with the mesh. The main idea is to modify the basis function over those triangles in which the interface cuts through so that the natural interface conditions are satisfied. The standard linear basis functions are used for other triangles. A level set function whose zero level set represent the interface is used. Numerical examples are also presented.
\end{abstract}

2000 AMS Mathematics Subject Classification: 65N06, 74B10, 74S30.

Keywords: Immersed finite element method, elasticity system, interface problems, Cartesian grids, jump conditions, level set method.

\section{Introduction}

In this paper, we develop a finite element method using a uniform Cartesian mesh for a two-phase elasticity system of equations of the form:

${ }^{*}$ Center for Research in Scientific Computation \& Department of Mathematics, North Carolina State University, Raleigh, NC 27695, email: zhilin@math.ncsu.edu

$\dagger$ Department of Mathematics, North Carolina State University, Raleigh, NC 27695, USA. Current addresss: Mathematics/CCS, Tulane University, 6823 St. Charles Ave., New Orleans, LA 70118, email: xyang3@math.ncsu.edu, xyang4@tulane.edu 


$$
\begin{array}{cl}
\nabla \cdot \boldsymbol{\sigma}+\boldsymbol{F}=0 & \text { in } \Omega, \\
{[\boldsymbol{u}]=0} & \text { on } \Gamma, \\
{[\boldsymbol{\sigma} \boldsymbol{n}]=0} & \text { on } \Gamma, \\
\boldsymbol{u}=\boldsymbol{u}_{\mathbf{0}} & \text { on } \partial \Omega,
\end{array}
$$

where $\boldsymbol{\sigma}$ is the stress tensor, $\boldsymbol{F}=\left(F_{1}, F_{2}\right)^{T}: \Omega \rightarrow \mathbb{R}^{2}$ is the body force, $\boldsymbol{u}=\left(u_{1}, u_{2}\right)^{T}$ is the displacement field, $\Gamma$ is a smooth interface that divides the domain $\Omega$ into two parts $\Omega^{+}$and $\Omega^{-}, \boldsymbol{n}=\left(n_{1}, n_{2}\right)^{T}$ is the unit normal vector of the interface $\Gamma$, pointing from the - phase to the + phase, and $\boldsymbol{u}_{\mathbf{0}}$ is a given vector-valued function that represents the displacement on the boundary $\partial \Omega$. Across the interface $\Gamma$, the physical parameters such as Young's modulus and Lamé constants have a finite discontinuity. For a function $v$, We use $[v]=$ $v^{+}-v^{-}$to denote the jump of $v$ across the interface $\Gamma$. The jump condition (1.2) means that $\boldsymbol{u}$ is continuous across the interface.

Multi-phase elasticity problems often arise in materials science, see for example, $[12,15]$. We refer the reader to $[1,6,9,17]$ for some applications and the references therein. However, solving such elasticity system is often difficult due to the arbitrary interface and the discontinuities in the coefficients and the gradient of the solution.

There exist several numerical methods for solving general elasticity problems that do not involve interfaces. Among them, the finite element method and the boundary integral or boundary element method appear to be very successful, cf. e.g., $[2,13,18]$ and the references therein. However, in treating moving interface problems, the use of fixed Cartesian grids often shows advantages in practical computations [7]. It is therefore desirable to develop new, efficient methods based on fixed Cartesian grids for the elasticity system with an interface.

In [17], a second order immersed interface method was developed for the elasticity system with an interface based on Cartesian grid. However, due to lack of the maximum principle, the stability of the method is still under investigation, and the linear solver for the finite difference equations may not converge satisfactorily. The goal of this paper is to develop an immersed finite element (IFE) for solving the two-phase elasticity system. The idea using a Cartesian mesh to solve a single elliptic interface problems can be found in [10, 11].

This paper is organized as follows: In Section 2, we derive the weak form of the elasticity system and the outline of the finite element method. In Section 3, we discuss how to constructs the basis functions for non-interface and interface elements. In Section 4, we explain how to use a the level set function to represent the interface. Several numerical examples are given in Section 5 . 


\section{Derivation of the weak form the elasticity sys- tem}

In this section, we derive the weak formulation of the elasticity system. We use more general boundary conditions (2.1) and (2.2) which replace (1.4)

$$
\begin{array}{ll}
\boldsymbol{\sigma} \boldsymbol{n}=\boldsymbol{t}, & \text { on } \partial \Omega_{1}, \\
\boldsymbol{u}=\boldsymbol{u}_{\mathbf{0}}, & \text { on } \partial \Omega_{2},
\end{array}
$$

where $\partial \Omega=\partial \Omega_{1} \cup \partial \Omega_{2}$. For convenience, we rewrite the strain and stress as vector forms

$$
\boldsymbol{\varepsilon}=\left[\begin{array}{c}
\varepsilon_{11} \\
\varepsilon_{22} \\
2 \varepsilon_{12}
\end{array}\right]=\left[\begin{array}{c}
\varepsilon_{x} \\
\varepsilon_{y} \\
\gamma_{x y}
\end{array}\right]=\left[\begin{array}{c}
\frac{\partial u}{\partial x} \\
\frac{\partial v}{\partial y} \\
\frac{\partial u}{\partial y}+\frac{\partial v}{\partial x}
\end{array}\right], \quad \boldsymbol{\sigma}=\left[\begin{array}{c}
\sigma_{11} \\
\sigma_{22} \\
\sigma_{12}
\end{array}\right]=\left[\begin{array}{c}
\boldsymbol{\sigma}_{x} \\
\boldsymbol{\sigma}_{y} \\
\boldsymbol{\tau}_{x y}
\end{array}\right] .
$$

If we introduce operator $\boldsymbol{A}$ as follows

$$
\boldsymbol{A}=\left[\begin{array}{cc}
\frac{\partial}{\partial x} & 0 \\
0 & \frac{\partial}{\partial y} \\
\frac{\partial}{\partial y} & \frac{\partial}{\partial x}
\end{array}\right]
$$

then the strain-displacement and stress-strain relations can be rewritten as

$$
\begin{gathered}
\boldsymbol{\varepsilon}=\left[\begin{array}{c}
\varepsilon_{x} \\
\varepsilon_{y} \\
\gamma_{x y}
\end{array}\right]=\left[\begin{array}{cc}
\frac{\partial}{\partial x} & 0 \\
0 & \frac{\partial}{\partial y} \\
\frac{\partial}{\partial y} & \frac{\partial}{\partial x}
\end{array}\right]\left[\begin{array}{l}
u \\
v
\end{array}\right]=\boldsymbol{A} \boldsymbol{u}, \\
\boldsymbol{\sigma}=\left[\begin{array}{c}
\boldsymbol{\sigma}_{x} \\
\boldsymbol{\sigma}_{y} \\
\boldsymbol{\tau}_{x y}
\end{array}\right]=[\boldsymbol{D}] \cdot \boldsymbol{\varepsilon}=[\boldsymbol{D}] \cdot\left[\begin{array}{c}
\boldsymbol{\varepsilon}_{x} \\
\boldsymbol{\varepsilon}_{y} \\
\boldsymbol{\gamma}_{x y}
\end{array}\right],
\end{gathered}
$$

where $[\boldsymbol{D}]$ is the elasticity matrix (or constitutive stress-strain matrix),

$$
[\boldsymbol{D}]=\left[\begin{array}{ccc}
\lambda+2 \mu & \lambda & 0 \\
\lambda & \lambda+2 \mu & 0 \\
0 & 0 & \mu
\end{array}\right]
$$

in which $\lambda$ and $\mu$ are Lamé constants. If we let $E$ be the Young's modulus, and $\nu$ be the Poisson's ratio, then we have

$$
\begin{gathered}
\mu=\frac{E}{2(1+\nu)}, \\
\lambda=\frac{E \nu}{1-\nu^{2}} \quad \text { (plane stress), } \quad \lambda=\frac{E \nu}{(1-2 \nu)(1+\nu)} \quad \text { (plane strain). }
\end{gathered}
$$


The equilibrium, constitutive and strain-displacement equations then become

$$
\begin{aligned}
\boldsymbol{A}^{T} \boldsymbol{\sigma} & =-\boldsymbol{F} \\
\boldsymbol{\sigma} & =[\boldsymbol{D}] \varepsilon \\
\boldsymbol{\varepsilon} & =\boldsymbol{A} \boldsymbol{u} .
\end{aligned}
$$

Eliminating $\boldsymbol{\sigma}$ and $\varepsilon$ gives the "displacement" formulations

$$
\boldsymbol{A}^{T}[\boldsymbol{D}] \boldsymbol{A} \boldsymbol{u}=-\boldsymbol{F} .
$$

Equation (2.9) will be used to derive the stiffness matrix.

\subsection{The variational form}

Let us consider the potential energy $\Pi$ of an elastic body. $\Pi$ is defined as the sum of the total strain energy $(U)$ and the work potential $(W P)$

$$
\Pi=\text { Strain energy }+ \text { Work Potential. }
$$

For linear elasticity materials, the strain energy per unit volume in the body is $\frac{1}{2} \boldsymbol{\sigma}^{T} \boldsymbol{\varepsilon}$. For a elastic body, the total energy $U$ is given by

$$
U=\frac{1}{2} \int_{\Omega} \boldsymbol{\sigma}^{T} \boldsymbol{\varepsilon} d \Omega=\frac{1}{2} \int_{\Omega} \boldsymbol{\varepsilon}^{T}[\boldsymbol{D}] \boldsymbol{\varepsilon} d \Omega .
$$

The work potential is given by

$$
W P=-\int_{\Omega} \boldsymbol{u}^{T} \boldsymbol{F} d \Omega-\int_{\Omega_{01}} \boldsymbol{u}^{T} \boldsymbol{t} d S .
$$

The total potential for the general elastic body is

$$
\Pi=\frac{1}{2} \int_{\Omega} \boldsymbol{\varepsilon}^{T}[\boldsymbol{D}] \boldsymbol{\varepsilon} d \Omega-\int_{\Omega} \boldsymbol{u}^{T} \boldsymbol{F} d \Omega-\int_{\Omega_{01}} \boldsymbol{u}^{T} \boldsymbol{t} d S .
$$

By the principle of minimum potential energy (cf. $[3,4,5,8,14]$ ), we obtain the following "variational form" or "weak form" for two-dimensional stress analysis ( $\boldsymbol{u}$ is an arbitrary displacement field):

$$
\int_{\Omega} \boldsymbol{\sigma}^{T} \boldsymbol{\varepsilon}(\boldsymbol{u}) d V=\int_{\Omega} \boldsymbol{u}^{T} \boldsymbol{f} d \Omega+\int_{\Omega_{01}} \boldsymbol{u}^{T} \boldsymbol{t} d S
$$

\subsection{The basic finite element equations}

We assume the domain $\Omega$ is a rectangle, but the interface $\Gamma$ can be arbitrary. We use a uniform triangulation regardless of the interface $\Gamma$. Therefore, the 
interface generally is not aligned with the edges of the triangulation. We need to find the basis functions for each right triangle of the partition. For each triangle, let $\left\{\boldsymbol{u}^{*}\right\}$ be the vector of the nodal displacements:

$$
\left\{\boldsymbol{u}^{*}\right\}=\left\{\begin{array}{llllll}
u_{1} & v_{1} & u_{2} & v_{2} & u_{3} & v_{3}
\end{array}\right\}
$$

The displacements at a point inside the triangle $\boldsymbol{u}$ can be determined in terms of the nodal displacements $\left\{\boldsymbol{u}^{*}\right\}$ and the basis, or shape, functions $\boldsymbol{N}$ :

$$
\boldsymbol{u}=[\boldsymbol{N}]\left\{\boldsymbol{u}^{*}\right\} .
$$

Strains and stresses can also be determined at nodal displacements:

$$
\begin{aligned}
& \boldsymbol{\varepsilon}=\boldsymbol{A u}=\boldsymbol{A}[\boldsymbol{N}]\left\{\boldsymbol{u}^{*}\right\}=[\boldsymbol{B}]\left\{\boldsymbol{u}^{*}\right\}, \\
& \boldsymbol{\sigma}=[\boldsymbol{D}] \boldsymbol{\varepsilon}=[\boldsymbol{D}][\boldsymbol{B}]\left\{\boldsymbol{u}^{*}\right\},
\end{aligned}
$$

where $[\boldsymbol{B}]=\boldsymbol{A}[\boldsymbol{N}]$ is called the displacement differentiation matrix, which can be obtained by differentiating displacements expressed through shape functions and nodal displacements. So we can obtain the following equilibrium equations for a finite element:

$$
\int_{\Omega}[\boldsymbol{B}]^{T}[\boldsymbol{D}][\boldsymbol{B}] d \Omega\{\boldsymbol{u}\}=\int_{\Omega}[\boldsymbol{N}]^{T} \boldsymbol{F} d x d y+\int_{\partial \Omega_{1}}[\boldsymbol{N}]^{T} \boldsymbol{t} d S .
$$

On each element, we have

$$
\int_{\Delta_{(e)}}[\boldsymbol{B}]^{T}[\boldsymbol{D}][\boldsymbol{B}] d \Omega\{\boldsymbol{u}\}=\int_{\Delta_{(e)}}[\boldsymbol{N}]^{T} \boldsymbol{F} d x d y+\int_{\partial \Omega_{1} \cap \Delta^{(e)}}[\boldsymbol{N}]^{T} \boldsymbol{t} d S .
$$

We need to know the basis function $\boldsymbol{N}$ for each element.

\section{Constructing the basis functions at interface triangles}

The triangles in our partition are classified into two categories: the interface triangles if the interface divides the triangle into two subsets, and non-interface triangles otherwise. For non-interface triangles, we use standard linear basis functions. However, for interface triangles, we use an undetermined coefficients method to determine the basis functions by enforcing the natural interface conditions. In the following discussions, we discuss how to construct such piecewise linear basis functions for interface triangular elements.

For a typical interface triangle $\triangle A B C$, let $B=\left(x_{1}, y_{1}\right), C=\left(x_{2}, y_{2}\right), A=$ $\left(x_{3}, y_{3}\right)$ be its vertices. Let $D=\left(x_{d}, y_{d}\right)$ and $E=\left(x_{e}, y_{e}\right)$ be the intersections 


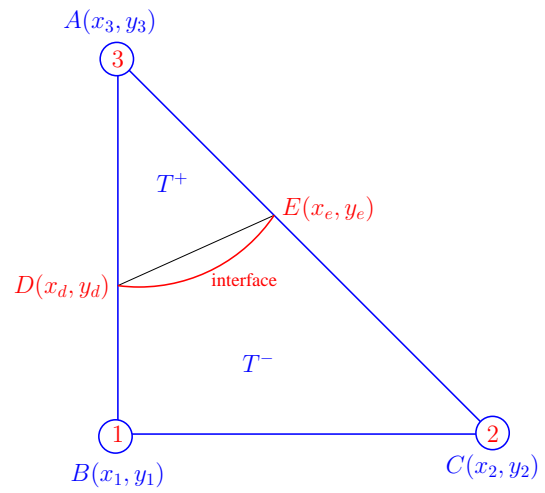

Figure 1: A typical interface triangle $\triangle A B C$.

of the interface and the edges of the triangle, see the sketch in Figure 1 for an illustration. For convenience, we denote $T^{+}$the region above the interface, and $T^{-}$the region below it.

Once the values $u, v$ at vertices $A, B, C$ of the element $T$ are specified, we construct the following piecewise linear function

$$
\begin{aligned}
& u(x, y)= \begin{cases}u^{+}(x, y)=a_{1}+a_{2} x+a_{3} y, & \text { if }(x, y) \in T^{+}, \\
u^{-}(x, y)=b_{1}+b_{2} x+b_{3} y, & \text { if }(x, y) \in T^{-}\end{cases} \\
& v(x, y)= \begin{cases}v^{+}(x, y)=c_{1}+c_{2} x+c_{3} y, & \text { if }(x, y) \in T^{+}, \\
v^{-}(x, y)=d_{1}+d_{2} x+d_{3} y, & \text { if }(x, y) \in T^{-},\end{cases}
\end{aligned}
$$

where $a_{i}$ 's, $b_{i}$ 's, $c_{i}$ 's, $d_{i}$ 's, $(i=1,2,3)$ are undetermined coefficients. The values of $u, v$ at the vertices $A, B, C$ are $u^{+}(A)=u_{1}, u^{-}(B)=u_{2}, u^{-}(C)=u_{3}$, $v^{+}(A)=v_{1}, v^{-}(B)=v_{2}, v^{-}(C)=v_{3}$. Therefore we have

$$
\begin{aligned}
& A\left(x_{3}, y_{3}\right):\left\{\begin{array}{l}
a_{1}+a_{2} x_{3}+a_{3} y_{3}=u_{3}, \\
c_{1}+c_{2} x_{3}+c_{3} y_{3}=v_{3} ;
\end{array}\right. \\
& B\left(x_{1}, y_{1}\right):\left\{\begin{array}{l}
b_{1}+b_{2} x_{1}+b_{3} y_{1}=u_{1}, \\
d_{1}+d_{2} x_{1}+d_{3} y_{1}=v_{1} ;
\end{array}\right. \\
& C\left(x_{2}, y_{2}\right):\left\{\begin{array}{l}
b_{1}+b_{2} x_{2}+b_{3} y_{2}=u_{2}, \\
d_{1}+d_{2} x_{2}+d_{3} y_{2}=v_{2} ;
\end{array}\right. \\
& D\left(x_{d}, y_{d}\right):\left\{\begin{array}{l}
a_{1}+a_{2} x_{d}+a_{3} y_{d}-b_{1}-b_{2} x_{d}-b_{3} y_{d}=0, \\
c_{1}+c_{2} x_{d}+c_{3} y_{d}-d_{1}-d_{2} x_{d}-d_{3} y_{d}=0 ;
\end{array}\right. \\
& E\left(x_{e}, y_{e}\right):\left\{\begin{array}{l}
a_{1}+a_{2} x_{e}+a_{3} y_{e}-b_{1}-b_{2} x_{e}-b_{3} y_{e}=0, \\
c_{1}+c_{2} x_{e}+c_{3} y_{e}-d_{1}-d_{2} x_{e}-d_{3} y_{e}=0 .
\end{array}\right.
\end{aligned}
$$


By the interface conditions,

$$
[\boldsymbol{\sigma n}]=\mathbf{0} \quad \text { on the interface } \Gamma,
$$

where $\boldsymbol{n}=\left[\begin{array}{l}n_{1} \\ n_{2}\end{array}\right]$ is the unit normal vector of the interface, which is perpendicular to $D E$, and

$$
\boldsymbol{\sigma} \boldsymbol{n}=\left[\begin{array}{c}
\sigma_{11} n_{1}+\sigma_{12} n_{2} \\
\sigma_{12} n_{1}+\sigma_{22} n_{2}
\end{array}\right] .
$$

In the component form, we have

$$
\begin{aligned}
& {\left.\left[(\lambda+2 \mu) \frac{\partial u}{\partial x} n_{1}+\lambda \frac{\partial v}{\partial y} n_{1}+\mu\left(\frac{\partial u}{\partial y}+\frac{\partial v}{\partial x}\right) n_{2}\right]\right|_{\Gamma}=0} \\
& {\left.\left[\mu\left(\frac{\partial u}{\partial y}+\frac{\partial v}{\partial x}\right) n_{1}+\lambda \frac{\partial u}{\partial x} n_{2}+(\lambda+2 \mu) \frac{\partial v}{\partial y} n_{2}\right]\right|_{\Gamma}=0}
\end{aligned}
$$

where (assuming the plane deformation)

$$
\mu=\frac{E}{2(1+\nu)}, \quad \text { and } \quad \lambda=\frac{E \nu}{(1+\nu)(1-2 \nu)} .
$$

If we use $[\boldsymbol{G}]$ to represent the coefficient matrix for the equations (3.3) (3.9), and use $\boldsymbol{X}$ to represent the vector formed by $a_{1}, a_{2}, a_{3}, b_{1}, b_{2}, b_{3}, c_{1}, c_{2}, c_{3}, d_{1}$, $d_{2}, d_{3}$, then $(3.3) \sim(3.9)$ can be rewritten as a matrix-vector form as follows:

$$
[\boldsymbol{G}] \boldsymbol{X}=[\boldsymbol{C}]\left\{\boldsymbol{u}^{*}\right\},
$$

where

$$
[\boldsymbol{C}]=\left[\begin{array}{cccccc}
0 & 0 & 0 & 0 & 1 & 0 \\
0 & 0 & 0 & 0 & 0 & 1 \\
1 & 0 & 0 & 0 & 0 & 0 \\
0 & 1 & 0 & 0 & 0 & 0 \\
0 & 0 & 1 & 0 & 0 & 0 \\
0 & 0 & 0 & 1 & 0 & 0 \\
0 & 0 & 0 & 0 & 0 & 0 \\
0 & 0 & 0 & 0 & 0 & 0 \\
0 & 0 & 0 & 0 & 0 & 0 \\
0 & 0 & 0 & 0 & 0 & 0 \\
0 & 0 & 0 & 0 & 0 & 0 \\
0 & 0 & 0 & 0 & 0 & 0
\end{array}\right], \quad\left\{\boldsymbol{u}^{*}\right\}=\left[\begin{array}{c}
u_{1} \\
v_{1} \\
u_{2} \\
v_{2} \\
u_{3} \\
v_{3}
\end{array}\right] .
$$

Solving 3.10 gives

$$
\boldsymbol{X}=[\boldsymbol{G}]^{-1}[\boldsymbol{C}]\left\{\boldsymbol{u}^{*}\right\}
$$


In other words, from (3.3) (3.7), and the interface conditions, we can express $a_{1}, a_{2}, a_{3}, b_{1}, b_{2}, b_{3}, c_{1}, c_{2}, c_{3}, d_{1}, d_{2}, d_{3}$ in terms of $u_{1}, v_{1}, u_{2}, v_{2}, u_{3}, v_{3}$, or $\left\{\boldsymbol{u}^{*}\right\}$. Therefore, $u^{+}, u^{-}, v^{+}, v^{+}$, can be expressed as

$$
\begin{aligned}
& u^{+}=\left(\overrightarrow{\boldsymbol{a}_{\mathbf{1}}}+x \overrightarrow{\boldsymbol{a}_{\mathbf{2}}}+y \overrightarrow{\boldsymbol{a}_{\mathbf{3}}}\right) \cdot\left\{\boldsymbol{u}^{*}\right\}, \\
& u^{-}=\left(\overrightarrow{\boldsymbol{b}_{\mathbf{1}}}+x \overrightarrow{\boldsymbol{b}_{\mathbf{2}}}+y \overrightarrow{\boldsymbol{b}_{\mathbf{3}}}\right) \cdot\left\{\boldsymbol{u}^{*}\right\}, \\
& v^{+}=\left(\overrightarrow{\boldsymbol{\boldsymbol { c } _ { \mathbf { 1 } }}}+x \overrightarrow{\boldsymbol{c}_{\mathbf{2}}}+y \overrightarrow{\boldsymbol{c}_{\mathbf{3}}}\right) \cdot\left\{\boldsymbol{u}^{*}\right\}, \\
& v^{-}=\left(\overrightarrow{\boldsymbol{d}_{\mathbf{1}}}+x \overrightarrow{\boldsymbol{d}_{\mathbf{2}}}+y \overrightarrow{\boldsymbol{d}_{\mathbf{3}}}\right) \cdot\left\{\boldsymbol{u}^{*}\right\},
\end{aligned}
$$

where $\overrightarrow{\boldsymbol{a}_{1}}=\left(a_{11}, a_{12}, a_{13}, a_{14}, a_{15}, \underline{a_{16}}\right)$ is the first row of matrix $[\boldsymbol{G}]^{-1}[\boldsymbol{C}]$ in (3.11), and so forth for $\overrightarrow{\boldsymbol{a}_{2}}, \cdots, \overrightarrow{\boldsymbol{d}_{3}}$. If we set

$$
\left[\begin{array}{l}
u \\
v
\end{array}\right]=\left[\begin{array}{llllll}
N_{11} & N_{12} & N_{13} & N_{14} & N_{15} & N_{16} \\
N_{21} & N_{22} & N_{23} & N_{24} & N_{25} & N_{26}
\end{array}\right]\left[\begin{array}{c}
u_{1} \\
v_{1} \\
u_{2} \\
v_{2} \\
u_{3} \\
v_{3}
\end{array}\right] \stackrel{\text { def }}{=}[\boldsymbol{N}]\left\{\boldsymbol{u}^{*}\right\},
$$

where $N_{i j}$ 's $(i=1,2, j=1,2, \cdots, 6)$ are piecewise linear functions, then we have

$$
[\boldsymbol{N}]=\left\{\begin{array}{l}
{\left[\begin{array}{l}
\overrightarrow{\boldsymbol{a}_{\mathbf{1}}}+x \overrightarrow{\boldsymbol{a}_{\mathbf{2}}}+y \overrightarrow{\boldsymbol{a}_{\mathbf{3}}} \\
\overrightarrow{\boldsymbol{c}_{\mathbf{1}}}+x \overrightarrow{\boldsymbol{c}_{\mathbf{2}}}+y \overline{\boldsymbol{c}_{\mathbf{3}}}
\end{array}\right], \quad+\text { side }} \\
{\left[\begin{array}{l}
\overrightarrow{\boldsymbol{b}_{\mathbf{1}}}+x \overrightarrow{\boldsymbol{b}_{\mathbf{2}}}+y \overrightarrow{\boldsymbol{b}_{\mathbf{3}}} \\
\overrightarrow{\boldsymbol{d}_{\mathbf{1}}}+x \overrightarrow{\boldsymbol{d}_{\mathbf{2}}}+y \overrightarrow{\boldsymbol{d}_{\mathbf{3}}}
\end{array}\right], \quad \text { side }}
\end{array}\right.
$$

Figures 2 and 3 show the mesh and contour plots of a pair of the basis functions in an interface triangles and in its entire support.

We then can compute the differentiation strain matrix $[\boldsymbol{B}]$ for interface elements:

$$
\begin{aligned}
& {[B]^{+}=A[N]=\left[\begin{array}{c}
\overrightarrow{a_{2}} \\
\overrightarrow{c_{3}} \\
\overrightarrow{a_{3}}+\overrightarrow{c_{2}}
\end{array}\right],} \\
& {[B]^{-}=A[N]=\left[\begin{array}{c}
\overrightarrow{b_{2}} \\
\overrightarrow{d_{3}} \\
\overrightarrow{b_{3}}+\overrightarrow{d_{2}}
\end{array}\right] .}
\end{aligned}
$$



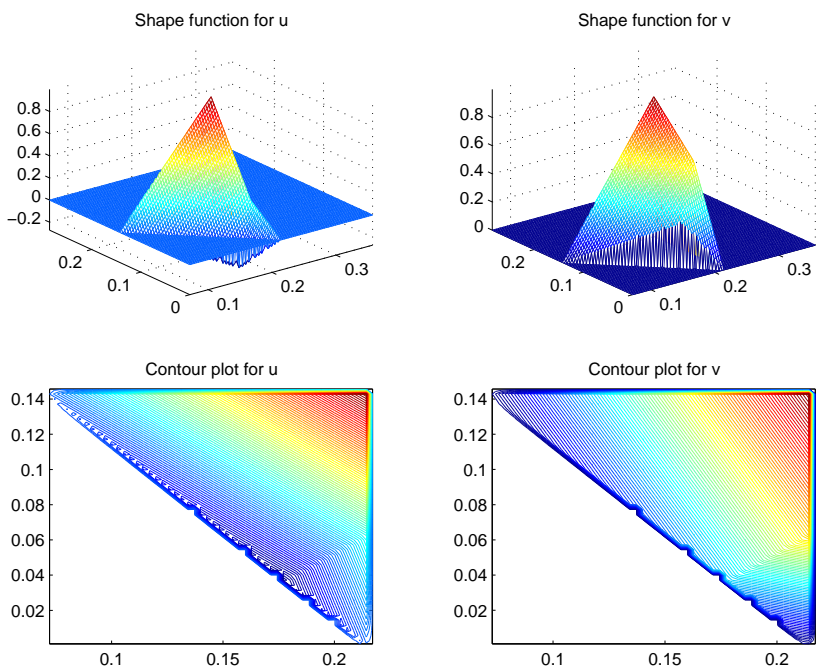

Figure 2: The mesh and contour plots of a pair of local basis functions $(u, v)$ over an interface triangle. The parameters are $\lambda^{+}=80, \lambda^{-}=160, \nu^{+}=0.35$, and $\nu^{-}=0.15$.

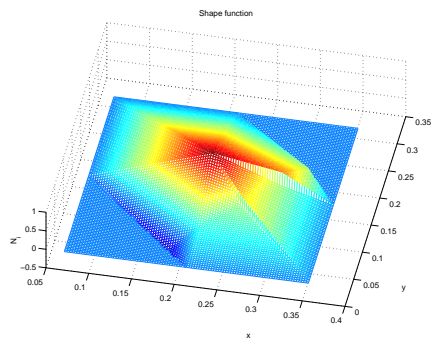

A global basis function

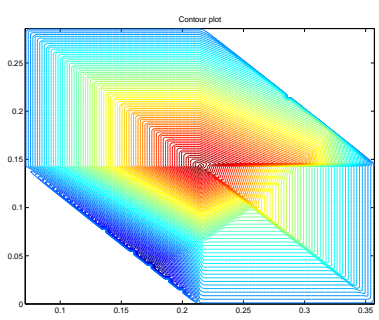

Contour plot

Figure 3: The mesh and contour plots of a global basis function $u$ on its support. The parameters are $\lambda^{+}=40, \lambda^{-}=90, \nu^{+}=0.35$, and $\nu^{-}=0.15$. 
Therefore, the local stiffness matrix is

$$
\begin{aligned}
{[\boldsymbol{k m}]=} & \int_{\Delta^{i e l}}[\boldsymbol{B}]^{T}[\boldsymbol{D}][\boldsymbol{B}] d x d y \\
= & \int_{\text {triangle }}\left[\boldsymbol{B}^{+}\right]^{T}\left[\boldsymbol{D}^{+}\right]\left[\boldsymbol{B}^{+}\right] d x d y \\
& +\int_{\text {other }}\left[\boldsymbol{B}^{-}\right]^{T}\left[\boldsymbol{D}^{-}\right]\left[\boldsymbol{B}^{-}\right] d x d y \\
= & {\left[\boldsymbol{B}^{+}\right]^{T}\left[\boldsymbol{D}^{+}\right]\left[\boldsymbol{B}^{+}\right] \cdot(\text { the area of the triangle }) } \\
& +\left[\boldsymbol{B}^{-}\right]^{T}\left[\boldsymbol{D}^{-}\right]\left[\boldsymbol{B}^{-}\right] \cdot(\text { the area of the other part }),
\end{aligned}
$$

which is also a constant and symmetric matrix.

\section{The interface and the level set method}

We use the zero level set of a Lipschitz continuous function $\phi(x, y)$ to represent the interface $\Gamma$. Usually $\phi(x, y)$ is chosen as the signed distance function. With the representation of a level set function, it is easy to compute the geometric information that is needed to construct the basis functions.

Theorem 4.1. Assume the coordinates of $A, B, C, D, E$ (see Figure 4) are $A\left(x_{i}, y_{j+1}\right), B\left(x_{i}, y_{j}\right), C\left(x_{i+1}, y_{j}\right), D\left(x_{D}, y_{D}\right), E\left(x_{E}, y_{E}\right)$. Then we have

$$
\begin{aligned}
x_{D} & =x_{i} \\
y_{D} & =y_{j}+\frac{\phi_{i, j} \Delta y}{\phi_{i, j}-\phi_{i, j+1}}+O\left((\Delta y)^{2}\right) \\
& =y_{j+1}-\frac{\phi_{i, j+1} \Delta y}{\phi_{i, j+1}-\phi_{i, j}}+O\left((\Delta y)^{2}\right), \\
x_{E} & =x_{i}+\frac{\Delta x \phi_{i, j+1}}{-\Delta x \phi_{x}(E)+\Delta y \phi_{y}(E)}+O(\Delta x \Delta y) \\
& =x_{i+1}+\frac{\Delta x \phi_{i+1, j}}{-\Delta x \phi_{x}(E)+\Delta y \phi_{y}(E)}+O(\Delta x \Delta y), \\
y_{E} & =y_{j}+\frac{\Delta y}{\Delta x}\left(x_{i+1}-x_{E}\right),
\end{aligned}
$$

where $\phi_{i, j+1}=\phi\left(x_{i}, y_{j+1}\right), \phi_{i, j}=\phi\left(x_{i}, y_{j}\right), \phi_{i+1, j}=\phi\left(x_{i+1}, y_{j}\right)$, and

$$
\begin{aligned}
\phi_{x}(E) & =\frac{\phi_{i, j}-\phi_{i+1, j}}{x_{i}-x_{i+1}}+O(\Delta x \Delta y), \\
\phi_{y}(E) & =\frac{\phi_{i, j+1}-\phi_{i, j}}{y_{j+1}-y_{j}}+O(\Delta x \Delta y) .
\end{aligned}
$$


The above theorem gives all the information that is needed to construct the basis function on an interface triangle.

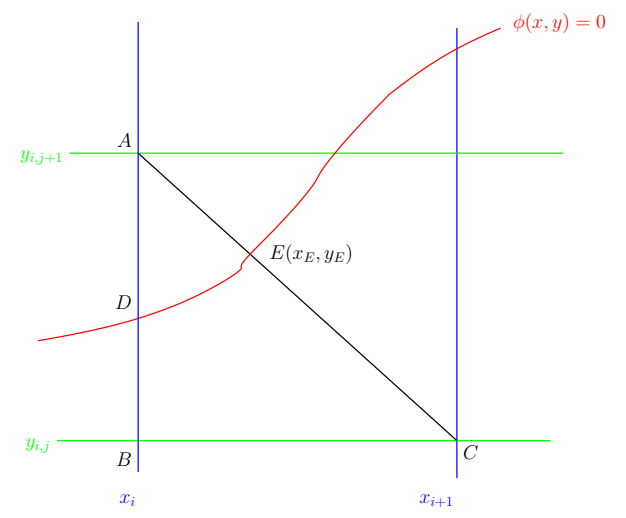

Figure 4: The geometric information of an interface triangles and the level set function representation.

The proof of the theorem is straightforward using the Taylor expansion at the intersections $D$ and $E$.

\section{$5 \quad$ Numerical examples}

In this section we present three numerical examples. Without loss of generality, the domain $\Omega$ is a rectangle $[-1,1] \times[-1,1]$, and the interface $\Gamma$ is $x^{2}+y^{2}=\frac{1}{4}$. In EXAMPLE 5.1 andEXAMPLE 5.2, we consider the system with zero body forces and homogeneous jumps conditions for displacements and traction across the interface. So the system is as follows,

$$
\begin{aligned}
\nabla \boldsymbol{\sigma} & =0, & & \text { in } \Omega^{+} \cup \Omega^{-}-\Gamma, \\
{[\boldsymbol{u}] } & =0, & & \text { on } \Gamma, \\
{[\boldsymbol{\sigma} \boldsymbol{n}] } & =0, & & \text { on } \Gamma, \\
\boldsymbol{u} & =\boldsymbol{u}_{\mathbf{0}}, & & \text { on } \partial \Omega,
\end{aligned}
$$

where $\boldsymbol{u}_{\mathbf{0}}=\left[u_{0}(x, y), v_{0}(x, y)\right]^{T}$. Since the analytic solution is not available, we compare the computed solutions obtained from the immersed finite element method with the solutions obtained from the immersed finite difference method developed in $[16,17]$ for example 5.1 to validate the IFEM method for the elasticity systems with interfaces. The immersed finite difference method developed in $[16,17]$ have been tested against the exact solution. EXAMPLE 5.2 is similar 
to EXAMPLE 5.1 except very large contrast (large jump ratio) of the physical parameters in which the finite difference method converges slower than the finite element element approach. In the last example ExAmpLE 5.3, we show a test result with non-zero source terms.

Example 5.1. In this example, the differential equations, interface and boundary conditions are exactly the same as that in Example 2.5.3 in [16].

$$
\begin{aligned}
& u_{0}(x, y)=-\frac{1}{10}\left(x^{2}+y^{2}\right)^{2}+\frac{1}{100} \ln \left(2 \sqrt{x^{2}+y^{2}}\right)-\frac{39}{160}, \\
& v_{0}(x, y)=\ln \left(1+x^{2}+3 y^{2}\right)+\sin (x y)-4 x^{2}-4 y^{2}+1 .
\end{aligned}
$$

The parameters are

$$
\begin{aligned}
& \nu^{-}=0.22, \quad \mu^{-}=200, \quad E^{-}=488, \\
& \nu^{+}=0.11, \quad \mu^{+}=60, \quad E^{+}=133.20 .
\end{aligned}
$$

Figure 5 and Figure 6 are the mesh and contour plots of the solution $(u, v)$ obtained by the IFEM method described in this paper using a 56 by 56 grid, which generates the system of size $6272 \times 6272$. From Figure 5 and Figure 6 , we can see they are almost identical to the figures by the finite difference method in Example 2.5.3 in [16]. The finite difference method is a little than the finite element method.
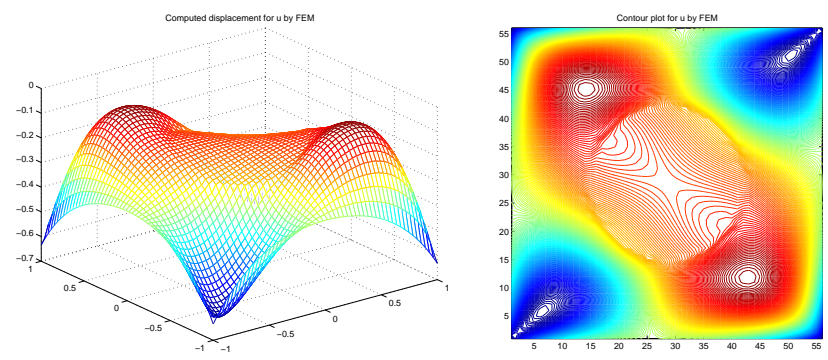

Figure 5: The mesh and contour plots of the computed solution $u$ of ExAMPLE 5.1 obtained by the immersed finite element method developed in this paper.

Example 5.2. In this example, we use the same differential equations, the jump conditions as that in ExAmple 5.1. But we use different boundary conditions and elasticity constants as follows:

$$
\begin{array}{lll}
\nu^{-}=0.02, & \mu^{-}=0.4902, & E^{-}=1, \\
\nu^{+}=0.49, & \mu^{+}=167.7852, & E^{+}=500
\end{array}
$$

and

$$
\begin{aligned}
& u_{0}(x, y)=\cos \left((x+1)^{2}\right)+y+1+\sin ((x+1)(y+1)) \\
& v_{0}(x, y)=(x+1)(y+1)+\sin (2(x+1)(y+1))
\end{aligned}
$$



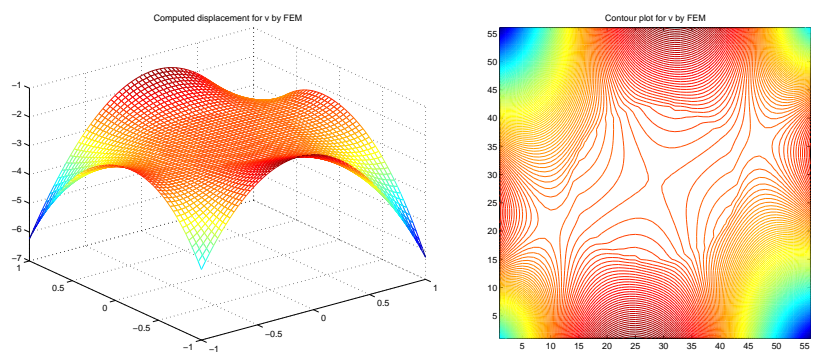

Figure 6: The mesh and contour plots of the computed solution $v$ of ExAMPLE 5.1 obtained by the immersed finite element method developed in this paper.

We have large jump ratio in the parameters. Figure (7) and Figure (8) are the mesh and contour plots for the displacements.

In this example the finite difference method takes much longer time to converge than the finite element method does. This is because the linear system of equations obtained from the finite element method is symmetric and has better condition number compared with that obtained from the finite difference approach.
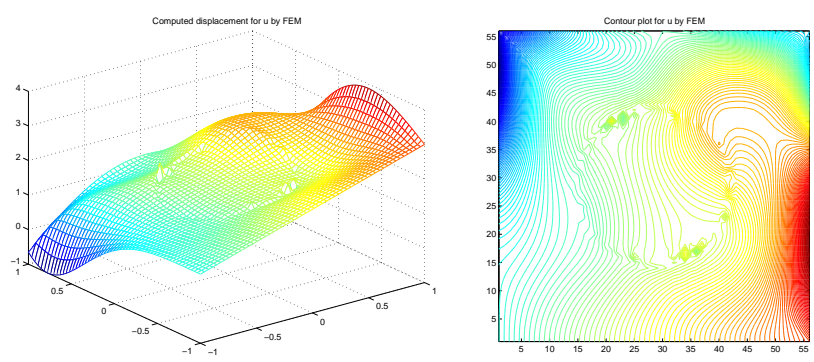

Figure 7: The mesh and contour plots of the computed solution $u$ of ExAMPLE 5.2 obtained by the immersed finite element method developed in this paper.

Example 5.3. In this example, the body forces are nonzero. The parameters for this example are $E^{+}=150, E^{-}=10, \nu^{+}=.2$ and $\nu^{-}=.24$. Figure (7) and Figure (8) are the mesh and contour plots of the displacements obtained from the finite element method developed in this paper.

From these examples, we see that the numerical results obtained from the immersed finite element method agree with the results by the finite difference method in $[16,17]$. But the system of linear equations obtained from the fi- 

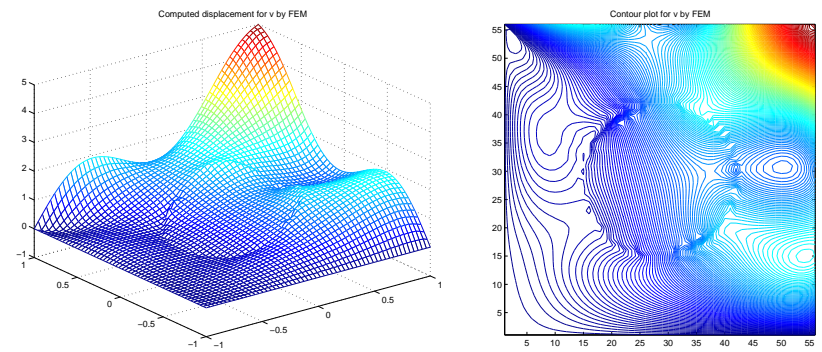

Figure 8: The mesh and contour plots of the computed solution $v$ of ExAMPLE 5.2 obtained by the immersed finite element method developed in this paper.
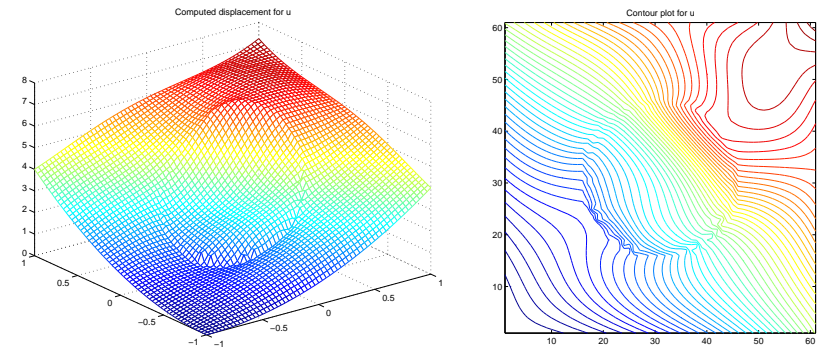

Figure 9: The mesh and contour plots of the computed solution $u$ of ExAMPLE 5.3 obtained by the immersed finite element method developed in this paper.
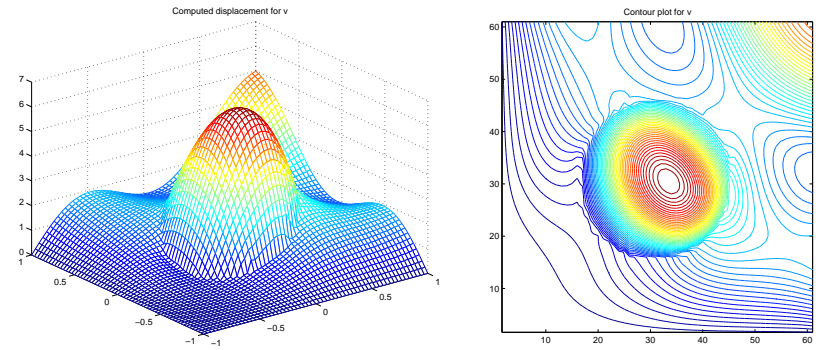

Figure 10: The mesh and contour plots of the computed solution $v$ of ExAMPLE 5.3 obtained by the immersed finite element method developed in this paper. 
nite element method has better condition than that obtained from the finite difference method.

\section{Conclusion and acknowledgments}

In this paper, we have developed the immersed finite element method based on a uniform Cartesian mesh for elasticity equations with discontinuous physical parameters across an interface. The interface does not have to be aligned with the mesh. The main idea is to modify the basis function over those triangles in which the interface cuts through so that the natural interface conditions are satisfied. The standard linear basis functions are used for other triangles. A level set function whose zero level set represent the interface is used. Numerical examples presented here show second order convergence for the new method.

The first author was partially supported by NSF grants DMS-0201094 and DMS0412654, and by an ARO grant 39676-MA.

\section{References}

[1] R. J. Asaro and W. A. Tiller. Surface morphology development during stress corrosion cracking: Part I: via surface diffusion. Metall. Trans., 3:1789-1796, 1972.

[2] I. Babuška and T. Strouboulis. The Finite Element Method and Its Reliability. Oxford University Press, 2001.

[3] Ti. R. Chandrupatla and A. D. Belegundu. Introduction to finite elements in engineering. Prentice Hall, Upper Saddle River, N.J., 1997.

[4] Y. C. Fung. Foundations of Solid Mechanics. Prentice Hall, Englewood Cliffs, N. J., 1965.

[5] Y. C. Fung and P. Tong. Classical and Computational Solid Mechanics. World Scientific, Singapore, 2001.

[6] M. A. Grinfeld. The stress driven rearrangement instability in ellastic crystals: mathematical models and physical manifestations. Nonlinear Sci., 3:35-83, 1993.

[7] T.Y. Hou. Numerical solutions to free boundary problems. Acta Numerica, pages 335-415, 1995. 
[8] K. H. Huebner, E. A. Thornton, and T. G. Byrom. The finite element method for engineers. A Wiley-Interscience Publication. John Wiley \& Sons Inc., New York, third edition edition, 1995.

[9] H.-J. Jou, H. Leo, and J. S. Lowengrub. Microstructrual evolution in inhomogeneous elastic media. J. Comp. Phy., 131(1):109-148, 1997.

[10] Z. Li. Immersed interface methods for moving interface problems. Numer. Algorithms, 14(4):269-293, 1997.

[11] Z. Li, T. Lin, and X. Wu. New Cartesian grid methods for interface problems using the finite element formulation. Numer. Math., 96(1):61-98, 2003.

[12] T. Mura. Micromechanics of defects in solids. Martinus Nijhoff Publishers, 1982.

[13] C. Pozrikidis. Boundary Element Methods. CRC Press, 2002.

[14] J. M. Steele. Applied Finite Element Modeling: Practical Problem Solving for Engineers. Mechanical engineering, 66. New York: M. Dekker, Inc, 1989.

[15] A. P. Sutton and R. W. Balluffi. Interfaces in Crystalline Materials. Oxford University Press, 1996.

[16] X. Yang. Immersed Interface Method for Elasticity Problems with Interfaces. PhD thesis, North Carolina State University, 2004.

[17] X. Yang, B. Li, and Z. Li. The immersed interface method for elasticity problems with interfaces. Dyn. Contin. Discrete Impuls. Syst. Ser. A Math. Anal., 10(5):783-808, 2003. Progress in partial differential equations (Pullman, WA, 2002).

[18] O. C. Zienkiewicz and R. L. Taylor. The finite element method. Vol. 2, Solid Mechanics. Butterworth-Heinemann, 2001. 